\title{
Oscillation Theorems for Certain Even Order Nonlinear Neutral Functional Differential Equations
}

\author{
Yan Zhao, Tongxing Li, Zhenlai Han* \\ School of Mathematical Sciences, \\ University of Jinan, P. R. China
}

\begin{abstract}
In this paper, some oscillation theorems of differential equation of the even order are established $(y(s)+P(s) y(\tau(s)))^{(n)}+Q(s) F(y(\sigma(s)))=0$, $t \geq t_{0}$, here $n \geq 2$ is even, $0 \leq P(s) \leq P_{0}<\infty$. The theorems extend and supplement some results in the literature. An example is investigated to illustrate our results.
\end{abstract}

Keywords-neutral functional differential equations; oscillation; even order.

\section{INTRODUCTION}

Neutral differential equations can be applied to numerous applications in engineering technique fields, physics, and so on. They are used for the research of distributed networks, for instance, see [1].

In the last decades, many researches have been made about the oscillation of solutions of neutral functional differential equations, we refer the reader to the papers [226].

In the paper, we consider the oscillatory property of nonlinear neutral differential equations of the even order

$$
\begin{gathered}
(y(t)+P(t) y(\tau(t)))^{(n)}+Q(t) F(y(\sigma(t)))=0, \\
t \geq t_{0},
\end{gathered}
$$

here $n \geq 2$ is even.

Below, we assume that

$\left(H_{1}\right) \quad Q$ and $P$ are continuous functions, $0 \leq P(t) \leq P_{0}<\infty$,

$$
Q(t)>0, t \in\left[t_{*}, \infty\right), t_{*} \geq t_{0}
$$

$\left(H_{2}\right) \quad \tau^{\prime}$ and $\sigma^{\prime}$ are continuous functions, $\sigma^{\prime}(t) \geq 0, \tau^{\prime}(t)=\tau_{0}>0, \sigma(t) \leq t$,

$\lim _{t \rightarrow \infty} \sigma(t)=\infty, \sigma(\tau(t))=\tau(\sigma(t)), \quad \tau_{0} \quad$ is a constant.

$\left(H_{3}\right) \quad F(u) / u \geq \alpha>0, F \quad$ is a continuous functions, $u \neq 0, \alpha$ is a constant.

Grammatikopoulos, Ladas and Meimaridou examined the oscillation of equations of second order

$$
[y(s)+P(s) y(s-\tau)]^{\prime \prime}+Q(s) y(s-\sigma)=0, s \geq s_{0},
$$

here $0 \leq P(s)<1$.

Baculíková and Lacková [3], Džurina and Hudáková [4] and Liu and Bai [14] examined neutral equations of the second order

$$
\begin{gathered}
\left(R(s)\left|Y^{\prime}(s)\right|^{\alpha-1} Y^{\prime}(s)\right)^{\prime}+Q(s)|y(\sigma(s))|^{\alpha-1} y(\sigma(s))=0, \\
s \geq s_{0},
\end{gathered}
$$

here $\quad Y(s)=y(s)+P(s) y(\tau(s)), \quad \sigma(s) \leq s$, $0 \leq P(s)<1$.

Han et al. [9] and $\mathrm{Ye}$ and $\mathrm{Xu}$ [22] considered quasilinear equations of the second order

$$
\begin{gathered}
\left(R(s) \psi(y(s))\left|Y^{\prime}(s)\right|^{\alpha-1} Y^{\prime}(s)\right)^{\prime}+Q(s) F(y(\sigma(s)))=0, \\
s \geq s_{0},
\end{gathered}
$$

here $Y(s)=y(s)+P(s) y(\tau(s)), \tau(s) \leq s, \sigma(s) \leq s$, $0 \leq P(s)<1$.

Meng and $\mathrm{Xu}$ [15] examined the oscillation of equations of even order

$\left[R(s)\left|(y(s)+P(s) y(s-\tau))^{(n-1)}\right|^{\alpha-1}(y(s)+P(s) y(s-\tau))^{(n-1)}\right]^{\prime}+Q(s) F(y(\sigma(s)))=0$, $s \geq s_{0}$, here $0 \leq P(s)<1, \sigma(s) \leq s$.

Zafer [23] examined oscillation of the equation

$$
\begin{gathered}
{[y(s)+P(s) y(\tau(s))]^{(n)}+F(s, y(s), y(\sigma(s)))=0,} \\
s \geq s_{0},
\end{gathered}
$$

here $1>P(s)>0$.

Zhang, Yan and Gao [24] investigated the oscillation of (1.1), where $0<P(s)<1$.

Clearly, the above oscillation theorems cannot be used to (1.1) when $1<P(s)$, it has few oscillation theorems about (1.1), when $1<P(t)$.

Han et al. [7, 8] studied equation (1.1) when $n=2$ and $0 \leq P(s) \leq P_{0}<\infty$. Li et al. [11] considered (1.1), when $\infty>P_{0} \geq P(s)>0$ and $\sigma(s) \geq \tau(s)$. 
Motivated by $[3,4,11,14]$, we will investigate equation (1), and offer new oscillation theorems for (1) where $\sigma(t) \leq \tau(t)$, by employing function $Y$, and operator $T$. Our results extend and supplement recent results given by [3] and [11], respectively.

Following [14], $\phi=\phi(t, s, l)$ belongs to the function class $Y$, denote by $\phi \in Y$, if $\phi$ continuous functions, which satisfies $\phi(t, s, l)>0, \quad \phi(t, t, l)=0$, $\phi(t, l, l)=0, \quad l<s<t, \partial \phi / \partial s$ is locally integrable with respect to $S$. By choosing $\phi$, it is possible to derive some oscillation theorems to another equations.

Define $T[\cdot ; l, t]$,

$$
T[g ; l, t]=\int_{l}^{t} \phi(t, s, l) g(s) \mathrm{d} s,
$$

$g \in C\left(\left[t_{0}, \infty\right), R\right), \quad t \geq s \geq l \geq t_{0} . \quad \varphi=\varphi(t, s, l)$ is defined by

$$
\frac{\partial \phi(t, s, l)}{\partial s}=\varphi(t, s, l) \quad \phi(t, s, l) .
$$

$T[\cdot ; l, t]$ is a linear operator,

$$
T\left[g^{\prime} ; l, t\right]=-T[g \varphi ; l, t],
$$

$g^{\prime}$ is continuous functions.

\section{MAIN RESULT}

In the section, some oscillation theorems of neutral functional differential equation of the even order (1) are derived. The following lemmas are needed.

Lemma 2.1. [16] Suppose $g \in C^{n}\left(\left[t_{0}, \infty\right), R^{+}\right)$, $g^{(n)}(s)$ is eventually of one sign for $s$, so there are $s_{x}>s_{0}$, integer $a, 0 \leq a \leq n$, with $n+a$ odd for $g^{(n)}(s) \leq 0$, or $n+a$ even for $g^{(n)}(s) \geq 0$, such that $a>0$ implies that $(-1)^{l+k} g^{k}(s)>0$, for $t>t_{x}$, $k=a, a+1, \cdots, a-1$, and $a \leq n-1$ implies that $g^{(k)}(s)>0$ for $s>s_{x}, k=0,1,2, \cdots, a-1$.

Lemma 2.2. [16] Suppose $g$ is as in Lemma 2.1, $g^{(n-1)}(s) g^{(n)}(s) \leq 0$ for $s>s_{x}, \quad$ so, for every $0<\lambda<1$, exists $M>0$, the following inequality holds, $g(\lambda s) \geq M s^{n-1}\left|g^{(n-1)}(s)\right|$.

Lemma 2.3. [15] Suppose $y$ is an eventually positive solutions of equations of the even order (1), then exists a $s_{1} \geq s_{0}$, the following inequality holds,

$$
B^{(n)}(s) \leq 0, B^{(n-1)}(s)>0, \quad B^{\prime}(s)>0, \quad B(s)>0,
$$

for $s \geq s_{1}$, where $B(s)=y(s)+P(s) y(\tau(s))$.

Theorem 2.1. If

$$
\int_{t_{0}}^{\infty} Q_{1}(s) \mathrm{ds}=\infty
$$

here $Q_{1}(s)=\min \{\alpha Q(s), \alpha Q(\tau(s))\}$, then every solution of functional differential equations of the even order (1) is oscillatory.

Proof. Suppose $y$ be a nonoscillatory solutions of equations of the even order $(1)$, then exists a $t_{1}, y(t) \neq 0$, $t \geq t_{1}$.

Assume that $y(t)>0, y(\sigma(t))>0, y(\tau(t))>0$, $t \geq t_{1}$, without loss of generality. From Lemma 2.3, exists a $t_{2} \geq t_{1}, \quad(2.1)$ holds. Using definition of $B(t)=y(t)+P(t) y(\tau(t))$ and applying (1), we get for sufficiently large $t$, the following inequality holds,

$B^{(n)}(t)+\alpha Q(t) y(\sigma(t))+\alpha P_{0} Q(\tau(t)) y(\sigma(\tau(t)))+\alpha P_{0} B^{(n)}(\tau(t)) \leq 0$.

So, the following inequality holds,

$$
B^{(n)}(t)+Q_{1}(t) B(\sigma(t))+\alpha P_{0} B^{(n)}(\tau(t)) \leq 0,
$$

here $Q_{1}(t)=\min \{\alpha Q(t), \alpha Q(\tau(t))\}$. Integrating from $t_{3}\left(\geq t_{2}\right)$ to $t$, and using $\tau^{\prime}(t) \geq \tau_{0}$, we obtain

$$
\begin{gathered}
\int_{t_{3}}^{t} Q_{1}(s) B(\sigma(s)) \mathrm{d} s \leq-\alpha P_{0} \int_{t_{3}}^{t} B^{(n)}(\tau(s)) \mathrm{d} s-\int_{t_{3}}^{t} B^{(n)}(s) \mathrm{d} s \\
\leq B^{(n-1)}\left(t_{3}\right)-B^{(n-1)}(t)+\frac{\alpha P_{0}}{\tau_{0}} B^{(n-1)}\left(\tau\left(t_{3}\right)\right)-\frac{\alpha P_{0}}{\tau_{0}} B^{(n-1)}(\tau(t)) .
\end{gathered}
$$

Since $B^{(n)}(t) \leq 0, B^{(n-1)}(t)>0, B^{\prime}(t)>0$, we have $\int_{t_{3}}^{\infty} Q_{1}(s) \mathrm{d} s<\infty$,

due to (8), which is a contradiction to (6).

Remark 2.1. Theorem 2.1 involves [3].

Theorem 2.2. Suppose $\sigma(t) \leq \tau(t)$, exist $\phi \in Y$, $k \in C^{1}\left(\left[t_{0}, \infty\right), R^{+}\right)$, such that for some $0<\lambda<1$, and for every $M>0$,

$$
\limsup _{t \rightarrow \infty} T\left[k(s) Q_{1}(s)-\frac{\left(1+\frac{\alpha P_{0}}{\tau_{0}}\right)\left(\varphi+\frac{k^{\prime}(s)}{k(s)}\right)^{2}}{4 \lambda M} \frac{k(s)}{\sigma^{n-2}(s) \sigma^{\prime}(s)} ; l, t\right]>0,
$$

here $Q_{1}(s)=\min \{\alpha Q(s), \alpha Q(\tau(s))\}, \quad T$ is defined by (2), $\varphi=\varphi(t, s, l)$ is defined as in (3), so, equations of the even order (1) is oscillatory. 
Proof. Suppose $y$ be a nonoscillatory solutions of equations of the even order (1), then exists $t_{1}$, such that $y(t) \neq 0, t \geq t_{1}$.

Let $\quad y(t)>0, \quad y(\sigma(t))>0, \quad y(\tau(t))>0$, $t \geq t_{1}$. There is a $t_{2} \geq t_{1}$, (5), (7) hold, by Lemma 2.3 and (1). We use Lemma 2.2 for $g=B^{\prime}$, exist $M>0, t_{3} \geq t_{2}$, the inequality holds,

$B^{\prime}(\lambda \sigma(t)) \geq M \sigma^{n-2}(t) B^{(n-1)}(\sigma(t)) \geq M \sigma^{n-2}(t) B^{(n-1)}(t),(10)$

So, we define

$$
Z(t)=\frac{k(t) B^{(n-1)}(t)}{B(\lambda \sigma(t))}
$$

So, $Z(t)>0$ and

$Z^{\prime}(t)=k^{\prime}(t) \frac{B^{(n-1)}(t)}{B(\lambda \sigma(t))}+k(t) \frac{B^{(n)}(t) B(\lambda \sigma(t))-\lambda B^{(n-1)}(t) B^{\prime}(\lambda \sigma(t)) \sigma^{\prime}(t)}{B^{2}(\lambda \sigma(t))}$

In view of $(10),(11),(12)$, we have

$Z^{\prime}(t) \leq k(t) \frac{B^{(n)}(t)}{B(\lambda \sigma(t))}+\frac{k^{\prime}(t)}{k(t)} Z(t)-\lambda M \frac{\sigma^{n-2}(t) \sigma^{\prime}(t)}{k(t)} Z^{2}(t)$.

Define another function

$$
A(t)=\frac{k(t) B^{(n-1)}(\tau(t))}{B(\lambda \sigma(t))} .
$$

So, $A(t)>0$ and

$$
\begin{aligned}
A^{\prime}(t) & =\frac{k^{\prime}(t) B^{(n-1)}(\tau(t))}{B(\lambda \sigma(t))} \\
+k(t) & \frac{B^{(n)}(\tau(t)) \tau^{\prime}(t) B(\lambda \sigma(t))-\lambda B^{(n-1)}(\tau(t)) B^{\prime}(\lambda \sigma(t)) \sigma^{\prime}(t)}{B^{2}(\lambda \sigma(t))} .
\end{aligned}
$$

From (10), (14), (15) and $\sigma(t) \leq \tau(t)$, we get

$$
\begin{aligned}
A^{\prime}(t) \leq & \frac{k(t) \tau^{\prime}(t) B^{(n)}(\tau(t))}{B(\lambda \sigma(t))}+\frac{k^{\prime}(t) A(t)}{k(t)} \\
& -\frac{\lambda M \sigma^{n-2}(t) \sigma^{\prime}(t) A^{2}(t)}{k(t)} .
\end{aligned}
$$

Then, by (13), (16), we have

$$
\begin{gathered}
Z^{\prime}(t)+\frac{\alpha p_{0}}{\tau^{\prime}(t)} A^{\prime}(t) \\
\leq \frac{k(t) B^{(n)}(t)}{B(\lambda \sigma(t))}+\frac{\alpha p_{0} k(t) B^{(n)}(\tau(t))}{B(\lambda \sigma(t))} \\
+\frac{k^{\prime}(t) Z(t)}{k(t)}-\frac{\lambda M \sigma^{n-2}(t) \sigma^{\prime}(t) Z^{2}(t)}{k(t)}
\end{gathered}
$$

$$
+\frac{\alpha p_{0}}{\tau^{\prime}(t)} \frac{k^{\prime}(t)}{k(t)} A(t)-\frac{\alpha p_{0}}{\tau^{\prime}(t)} \lambda M \frac{\sigma^{n-2}(t) \sigma^{\prime}(t)}{k(t)} A^{2}(t) .
$$

From (7), the following inequality holds,

$$
\begin{gathered}
Z^{\prime}(t)+\frac{\alpha p_{0}}{\tau_{0}} A^{\prime}(t) \leq-k(t) Q(t)+\frac{k^{\prime}(t)}{k(t)} Z(t) \\
-\frac{\lambda M \sigma^{n-2}(t) \sigma^{\prime}(t) Z^{2}(t)}{k(t)}+\frac{\alpha p_{0}}{\tau_{0}} \frac{k^{\prime}(t) A(t)}{k(t)} \\
-\frac{\alpha p_{0}}{\tau_{0}} \lambda M \frac{\sigma^{n-2}(t) \sigma^{\prime}(t)}{k(t)} A^{2}(t)
\end{gathered}
$$

By $T[\cdot ; l, t]$ into (17), we have

$$
\begin{gathered}
T\left[Z^{\prime}(s)+\frac{\alpha p_{0} A^{\prime}(s)}{\tau_{0}} ; l, t\right] \leq \\
T\left[-k(s) Q(s)+\frac{k^{\prime}(s) Z(s)}{k(s)}-\lambda M \frac{\sigma^{n-2}(s) \sigma^{\prime}(s) Z^{2}(s)}{k(s)}+\frac{\alpha p_{0}}{\tau_{0}} \frac{k^{\prime}(s) A(s)}{k(s)}-\frac{\alpha p_{0} \lambda M}{\tau_{0}} \frac{\sigma^{n-2}(s) \sigma^{\prime}(s) A^{2}(s)}{k(s)} ; l, t\right] .
\end{gathered}
$$

By (4), the inequality of about, the following inequality holds,

$$
\begin{gathered}
T[k(s) Q(s) ; l, t] \leq T\left[\left(\varphi+\frac{k^{\prime}(s)}{k(s)}\right) Z(s)\right. \\
-\lambda M \frac{\sigma^{n-2}(s) \sigma^{\prime}(s)}{k(s)} Z^{2}(s) \\
\left.+\frac{\alpha P_{0}}{\tau_{0}}\left(\varphi+\frac{k^{\prime}(s)}{k(s)}\right) A(s)-\frac{\alpha P_{0}}{\tau_{0}} \lambda M \frac{\sigma^{n-2}(s) \sigma^{\prime}(s)}{k(s)} A^{2}(s) ; l, t\right] .
\end{gathered}
$$

So, by (18), the following inequality holds,

$$
T[k(s) Q(s) ; l, t]
$$

$$
\leq T\left[\left(\frac{\left(\varphi+\frac{k^{\prime}(s)}{k(s)}\right)^{2}+\frac{\alpha P_{0}}{\tau_{0}}\left(\varphi+\frac{k^{\prime}(s)}{k(s)}\right)^{2}}{4 \lambda M}\right) \frac{k(s)}{\sigma^{n-2}(s) \sigma^{\prime}(s)} ; l, t\right],
$$

then,

$$
T\left[k(s) Q(s)-\frac{\left(1+\frac{\alpha P_{0}}{\tau_{0}}\right)\left(\varphi+\frac{k^{\prime}(s)}{k(s)}\right)^{2}}{4 \lambda M} \frac{k(s)}{\sigma^{n-2}(s) \sigma^{\prime}(s)} ; l, t\right] \leq 0 .
$$

The following results are obtained,

$$
\limsup _{t \rightarrow \infty} T\left[k(s) Q(s)-\frac{\left(1+\frac{\alpha P_{0}}{\tau_{0}}\right)\left(\varphi+\frac{k^{\prime}(s)}{k(s)}\right)^{2}}{4 \lambda M} \frac{k(s)}{\sigma^{n-2}(s) \sigma^{\prime}(s)} ; l, t\right] \leq 0,
$$

which contradicts (9).

Remark 2.2. Theorem 2 extends [3].

Remark 2.3. The results got in this paper complement the results got in [11].

Example 2.1 Investigate the even order equations

$$
[y(s)+2 y(s+\pi)]^{(n)}+y\left(s-\frac{n \pi}{2}\right)=0 .
$$

Let

$$
Q(s)=1, P(s)=2, \quad \sigma(s)=s-\frac{n \pi}{2}, \tau(s)=s+\pi,
$$


Then, we have every solution of the even order neutral equations (19) is oscillatory by Theorem 2.1. In fact, $y(s)=\sin s$ is an oscillatory solution of (19).

\section{ACKNOWLEDGEMENTS}

This research is supported by the NSF of China (61374074), and supported by NSF of Shandong Provincial (ZR2012AM009, ZR2013AL003).

*Corresponding author: Zhen-Lai Han, e-mail: hanzhenlai@163.com.

\section{REFERENCES}

[1] Hale, J. K. Theory of functional differential equations, Springer-Verlag, New York, 1977.

[2] Agarwal, R. P., Grace, S. R., O'Regan, D. Oscillation theory for difference and functional differential equations, Kluwer Academic Publishers, Dordrecht, 2000.

[3] Baculíková, B., Lacková, D. Oscillation criteria for second order retarded differential equations. Studies of the University of Zilina, Mathematical Series, 20, pp.11-18, 2006.

[4] Džurina, J., Hudáková, D. Oscillation of second order neutral delay differential equations. Math. Bohem., 134, pp. 31-38, 2009.

[5] Grace, S. R. Oscillation theorems for nonlinear differential equations of second order. J. Math. Anal. Appl., 171, pp.220-241, 1992.

[6] Grammatikopoulos, M. K., Ladas, G., Meimaridou, A. Oscillation of second order neutral delay differential equation. Rat. Mat., 1, pp. 267-274, 1985.

[7] Han, Z. L., Li, T. X., Sun, S. R., Sun, Y. B. Remarks on the paper [Appl. Math. Comput. 207 (2009) 388-396]. Appl. Math. Comput., 215, pp.3998-4007, 2010.

[8] Han, Z. L., Li, T. X., Sun, S. R., Chen, W. S. On the oscillation of second-order neutral delay differential equations. Adv. Differ. Equ., 2010, pp. 1-8, 2010.

[9] Han, Z. L., Li, T. X., Sun, S. R., Chen, W. S. Oscillation criteria for second-order nonlinear neutral delay differential equations. Adv. Differ. Equ., 2010, 1-23 (2010).

[10] Karpuz, B., Manojlović, J. V., Öcalan, Ö, Shoukaku, Y. : Oscillation criteria for a class of second-order neutral delay differential equations. Appl. Math. Comput., 210, 303-312 (2009).
[11] Li, T. X., Han, Z. L., Zhao, P., Sun, S. R. Oscillation of even-order neutral delay differential equations. Adv. Differ. Equ., 2010, pp.1-9, 2010.

[12] Li, H. J., Yeh, C. C. Oscillation criteria for second order neutral delay equations. Comput. Math. Appl., 36, pp.123-132, 1998.

[13] Lin, X. Y., Tang, X. H. Oscillation of solutions of neutral differential equations with a superlinear neutral term. Appl. Math. Lett., 20, pp.1016-1022, 2007.

[14] Liu, L. H., Bai, Y. Z. New oscillation criteria for second-order nonlinear neutral delay differential equations. J. Comput. Appl. Math., 231, pp.657-663, 2009.

[15] Meng, F. W., Xu, R. Oscillation criteria for certain even order quasilinear neutral differential equations with deviating arguments. Appl. Math. Comput., 190, pp.458-464, 2007.

[16] Philos, Ch. G. A new criteria for the oscillatory and asymptotic behavior of delay differential equations. Bull. Acad. Pol. Sci. Ser. Sci. Mat., 39, pp.61-64, 1981.

[17] Rath, R. N., Misra, N., Panhy, L. N. Oscillatory and asymptotic behaviour of a nonlinear second order neutral differential equations. Math. Slovaca, 57, pp.157-170, 2007.

[18] Şahiner, Y. On oscillation of second order neutral type delay differential equations. Appl. Math. Comput., 150, pp.697-706, 2004.

[19] Sun, Y. G., Meng, F. W. Note on the paper of Džurina and Stavroulakis. Appl. Math. Comput., 174, pp.1634-1641, 2006.

[20] Xu, R., Meng, F. W. Oscillation criteria for second order quasi-linear neutral delay differential equations. Appl. Math. Comput., 192, pp.216-222, 2007.

[21] Xu, Z. T., Liu, X. X. Philos-type oscillation criteria for Emden-Fowler neutral delay differential equations. J. Comput. Appl. Math., 206, pp.1116-1126, 2007.

[22] Ye, L. H., Xu, Z. T. Oscillation criteria for second order quasilinear neutral delay differential equations. Appl. Math. Comput., 207, pp.388-396, 2009.

[23] Zafer, A. Oscillation criteria for even order neutral differential equations. Appl. Math. Lett., 11, pp.21-25, 1998.

[24] Zhang, Q. X., Yan, J. R., Gao, L. Oscillation behavior of even-order nonlinear neutral differential equations with variable coefficients. Comput. Math. Appl., 59, pp.426-430, 2010.

[25] Han, Z. L., Li, T. X., Sun, S. R., Chen, W. S. Oscillation of second order quasilinear neutral delay differential equations. J. Appl. Math. Comput., 40, pp.143-152, 2012.

[26] Li, T. X., Han, Z. L., Zhang, C. H., Sun, S. R. On the oscillation of second-order Emden-Fowler neutral differential equations. J. Appl. Math. Comput., 37(1-2), pp.601-610, 2011. 